\title{
EMERGENCY RADIATION SURVEY DEVICE ONBOARD THE UAV
}

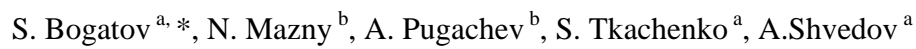 \\ a Nuclear Safety Institute, 115191 Moskow, B Tulskaya str. 52, Russia - (sbg, tsa, sham@ibrae.ac.ru) \\ b SPC ASPECT, 141980 Moscow region, Dubna, Sakharova str. 6, Russia - (nikitos, pgv@aspect.jinr.ru)
}

KEY WORDS: Unmanned Aerial Vehicle, Radiation Survey, Radionuclide, Dose Rate, Surface Contamination, Point Source

\begin{abstract}
:
Radiation survey device (RSD) on the base of unmanned aerial vehicle (UAV) was developed as an equipment of rescue forces for radiation situation reconnaissance in case of emergency. RSD is multi range radiometer with spectrometer functions capable to work within gamma ray fields of dose rate $10^{-7}-10^{-1}$ Sievert per hour. UAV md4-1000 (Microdrones GmbH, Germany) was selected as the RSD carrier as a reliable vehicle with appropriate properties. Short description of RSD, UAV and developed software features as well as sensitivity assessments for different radiation sources are presented.
\end{abstract}

\section{INTRODUCTION}

Now there is a tendency to optimize an amount and equipment of the rescue forces with regard to severity and probability of the accidents. It is especially important for radiation accidents those are very rare events but demanding involvement of huge mitigation resources in case of occurrence. In this case permanent ready large rescue teams for radiation situation survey became very expensive. Specialized, high efficacy survey devices served by few personnel may be a better solution.

This type of devices is urgent to survey places of accidents immediately. One of possible solution is mounting radiation survey devices (RSD) capable to combine radiometric and spectroscopic measurements within high radiation levels, onboard of unmanned aerial vehicles (UAV). This solution makes it possible to achieve key aerial survey advantages, that is workability in high radiation field without hazardous operator irradiation, survey of large areas for a short time (representative measurements), survey of hard available areas (ruins, fire etc) and radioactive plume studies.

\section{CHOICE OF UAV}

First requirements to the UAV were proved reliability in operation and easy control by non professional pilot. The carrier must be serial and have acceptable price.

Regarding necessary flight distance being of several kilometers and usual lack of "direct vision" condition between the UAV and operator at that distance, capability of autonomous flight along pre-defined path was required.

To correct the flight path depending on current radiation measurement results, the UAV must have data transfer channel to ground base station. This channel has to be able to include specific radiation measurements data into general telemetry information flux of the UAV.

One more necessary feature was the UAV equipment by photo camera and coordinate definition for the pictures have been made. This requirement is caused by difficulties of aerial data interpretation when radioactive source is shielded by the some buildings or naturally occurred radionuclides contribution from adjacent structures becomes significant. In this case knowing the picture of the surface beneath the UAV is necessary.

Obvious requirement is definition of geographic coordinates during the flight.

Payload mass limitation of $1.2 \mathrm{~kg}$ for md4-1000 was not critical for the tasks planned. Within high level radiation fields, large volume and mass of the sensors are not necessary (but sometimes harmful) and the measurements can be carried out by compact devices.

All these reasons substantiated the choice of md4-1000 (Fig. 1) as a carrier for RSD developed.

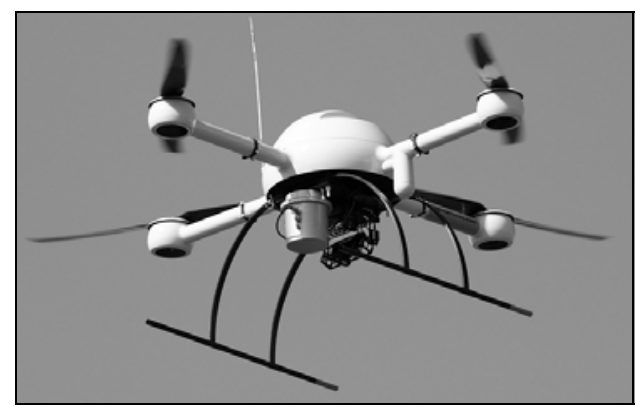

Figure 1. UAV md4-1000 with RSD and photo camera as a payload

\section{APPROACHES TO THE RSD DEVELOPMENT}

One of the basic RSD requirements were operability in large gamma dose rate interval $\left(10^{-7}-10^{-1}\right.$ Sievert/hour $)$ for the time of single measurement of 1 second and correspondence to size and mass UAV payload limitation.

Pilot RSD example (Fig. 2) was developed and manufactured by Scientific and Industrial Centrum "ASPECT" (Dubna, Russia).

The device is situated within thin-wall aluminum shell, spectrometer on the base of $\mathrm{NaJ}(\mathrm{Tl})$ crystal and two GeigerMuller counters are used as the radiation sensors. Several RSD technical parameters are shown in Table 1.

\footnotetext{
* Corresponding author.
} 
Spectrometric measurements are used within dose rate range from $10^{-7} \mathrm{~Sv} / \mathrm{h}$ to $10^{-4} \mathrm{~Sv} / \mathrm{h}$, Geiger-Muller counters cover dose rate range $10^{-4}-10^{-1} \mathrm{~Sv} / \mathrm{h}$.

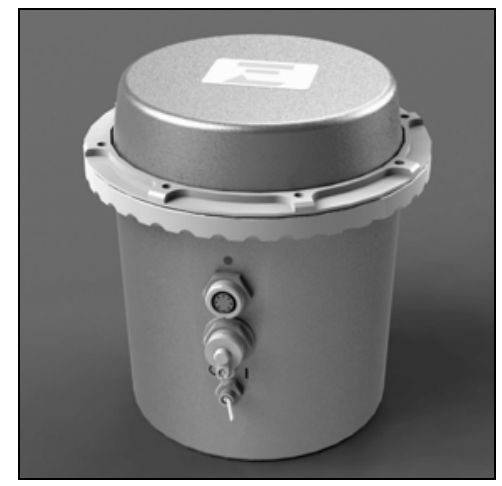

Figure 2. RSD exterior

RSD uses power supply and data transfer channel of the UAV. Having been connected to UAV and turned on, RSD automatically starts the measurements with time interval of $1 \mathrm{~s}$ and simultaneously transfers obtained count rates of the spectrometer and Geiger-Muller counter to the UAV controller. Spectra obtained for every second and some attributive information including GPS coordinates from the UAV are stored in the RSD flash memory.

UAV data parcel is transferred to ground base station four times a second. Only one payload identificator and correspondent value can be included in the drone data parcel, so only four payload parameters per second can be transferred through this channel. To increase RSD data transfer reliability, it was decided to send spectrometer and Geiger-Muller count rates twice a second. Data losses of only 5\% were achieved applying this approach.

\begin{tabular}{|l|c|}
\hline Parameter & Value \\
\hline Energy range, MeV & 0,05 - 3 \\
Relative energy resolution at & 7.5 \\
$662 \mathrm{keV}\left({ }^{137} \mathrm{Cs}\right), \%$ & \pm 1 \\
Calibration stability for $8 \mathrm{~h}$ & $1,5 \cdot 10^{5}$ \\
operation, \% & 230 \\
Maximal spectral count rate, $\mathrm{s}^{-1}$ & from $10^{-7}$ to $10^{-1}$ \\
Number of spectrum channels & 430 \\
Dose rate measurable, Sv/h & $\mathrm{RS}-232 \mathrm{TTL}$ \\
Scintillation detector sensitivity & 38400 \\
(for Cs-137), ${ }^{-1} / \mu$ Sv & from 0 to +50 \\
Communication cable interface & 0,55 \\
Data transfer rate, kbit/s & $111 \times 90 \times 126$ \\
Working temperature, ${ }^{0} \mathrm{C}$ & \\
Mass, kg & \\
Dimension, mm &
\end{tabular}

Table 1. RSD technical parameters

\section{RSD SOFTWARE}

RSD software has been developed for data acquisition and visualization during the flight and more accurate data processing after the UAV landing.

RSD software receives the measurement data from mdCockpit program during the flight and displays it at the map.
Measurement results appear at the map in real time (approximately every second) as colored points. Point position corresponds to the measurement coordinate, color corresponds to gamma radiation dose rate at the ground. Point color is taken from gradient scale (low right corner of the map in Fig. 3) which boundaries can be chosen by the user.

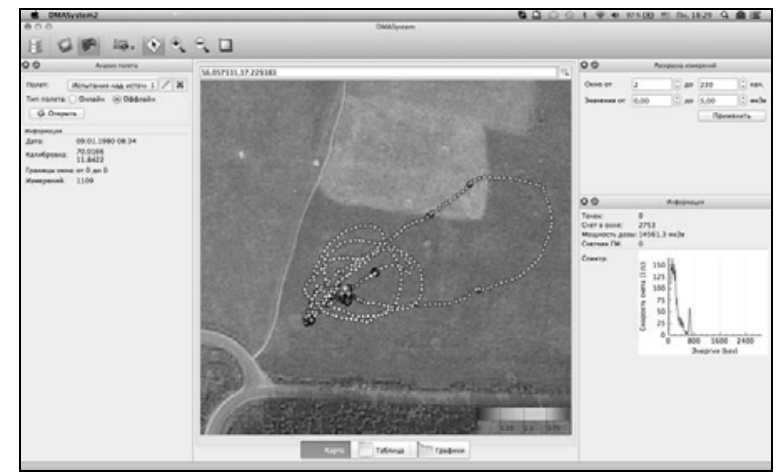

Figure 3. One of the working windows of the RSD software measurement data display at the map

RSD data are written in temporal data base including spectral and counter rates with appropriate coordinates. This data may be used in case of the UAV loss, but usually data stored in the RSD memory are used as more accurate and free from missing values.

Post-flight data processing includes as follows:

- display of the measurement point attributive information at the mouse click on point at the map;

- calculation and display of the spectrum being the summary ones over points selected at the map;

- calculation of average dose rate over points selected at the map;

- calculation for summary spectrum the natural background contribution;

- algorithm for volume contamination with uniform or exponential activity depth profile calculation;

- algorithm to search for point source location and activity calculation.

\section{RSD SENSITIVITY TO DETECT RADIATION ANOMALIES}

Having detector gamma-ray registration efficiency and RSD parameters for background gamma radiation, it is possible to assess expectable parameters for detection of radiation anomalies. Detector efficiency has been obtained by MCNP calculations (Bogatov, 2012), background spectra from test flights were used for background count rate estimation.

Performed assessments showed that ${ }^{137} \mathrm{Cs}$ point source with activity of $10^{9} \mathrm{~Bq}$ can be detected at the flight heights less then $50 \mathrm{~m}$ if detection criterion of background count rate increase by one standard deviation value is used.

${ }^{137} \mathrm{Cs}$ surface contamination of $10^{5} \mathrm{~Bq} / \mathrm{m}^{2}$ according to selected criterion can be clearly detected at heights less then $100 \mathrm{~m}$.

\section{CONCLUSION}

Md4-1000 proved to be reliable as a RSD carrier, appropriate RSD was successfully manufactured and tested.

RSD is multi range radiometer with spectrometer functions capable to work within gamma ray fields of dose rate $10^{-7}-10^{-1}$ 
Sievert per hour. RSD sensitivity for ${ }^{137}$ Cs point source detection (by full absorption peak) is assessed to be about $10^{9} \mathrm{~Bq}$ from the flight heights less than $70 \mathrm{~m}$. Surface contamination of $10^{5} \mathrm{~Bq} / \mathrm{m}^{2}$ of ${ }^{137} \mathrm{Cs}$ may be surely identified from height of $100 \mathrm{~m}$ above the ground.

Flight tests and software improvement are being performed.

\section{REFERENCES}

Bogatov, S., 2012. Mobile radiation survey device on the base of unmanned aerial vehicle md4-1000. Special equipment, 6, pp. 16-22. 